\title{
TERAPIA OCUPACIONAL ¿PARA TODOS? SU EXCLUSIÓN PARCIAL DE FONASA EN USUARIOS PEDIÁTRICOS CON PARÁLISIS CEREBRAL EN LA REGIÓN DE MAGALLANES, CHILE
}

\author{
OCCUPATIONAL THERAPY FOR ALL? IT'S PARTIAL EXCLUSION OF FONASA IN \\ USERS WITH CEREBRAL PALSY IN THE REGION OF MAGALLANES, CHILE
}

\section{Paulina Almonacid Paillaman', Decxye Figueroa Vergara², Geraldine Söllner González ${ }^{3}$ y Diego Solsona Cisternas ${ }^{4}$}

\begin{abstract}
RESUMEN
El siguiente artículo establece la importancia y necesidad de incorporar totalmente las diversas prestaciones de Terapia Ocupacional (en adelante T.O) al catálogo del Fondo Nacional de Salud (en adelante FONASA) considerando a esta profesión como prestadora de servicios de rehabilitación transitoria y permanente en usuarios con diferentes diagnósticos y/o enfermedades que se prolongan durante todo el ciclo vital. El objetivo general fue "conocer los significados que tienen los familiares de usuarios con necesidad de rehabilitación permanente con respecto a la situación de que algunos servicios o prestaciones profesionales asistenciales de T.O no se encuentren incluidos en el sistema FONASA". Esta investigación se basa en los discursos de familiares (Padres-Madres) de niños/as con parálisis cerebrales (en adelante PC) de una agrupación perteneciente a la región de Magallanes, donde se plantea desde una metodología cualitativa (aplicación de entrevistas semi-estructuradas). Los resultados sugieren que los familiares de niños/as con PC, expresan conocer el rol e importancia de un/a T.O en el proceso de rehabilitación de sus hijos, enfatizando en que es necesario que el Estado proporcione los recursos necesarios, para que las prestaciones de los profesionales puedan ser más accesibles.

Finalmente, este artículo no solo intenta validar las intervenciones de los T.O para incorporarlos al catálogo de prestaciones de FONASA, sino que rescata las percepciones subjetivas de quienes dependen de estas intervenciones, y que se encuentran, en algún sentido, imposibilitados de ejercer su derecho a una salud pública accesible a sus necesidades, desde un enfoque de derechos humanos.
\end{abstract}

PALABRAS CLAVES

Terapia Ocupacional; Fondo nacional de salud, Salud Pública; Parálisis Cerebral; Rehabilitación, Derechos Humanos.

1 Licenciada en ciencia de la ocupación, Universidad de Magallanes, paulialmonacid18@gmail.com

2 Licenciada en ciencia de la ocupación, Universidad de Magallanes, f-figueroa1@hotmail.com

3 Licenciada en ciencia de la ocupación, Universidad de Magallanes. geraldine.sollnerg@gmail.com

4 Doctorando de ciencias sociales en estudios territoriales por la Universidad de Los Lagos, Licenciado en sociología, sociólogo y Magister en investigación social y desarrollo por la Universidad de Concepción, Docente adjunto e investigador del Departamento de Terapia ocupacional, Universidad de Magallanes, diego.solsona@umag.cl 


\section{ABSTRACT}

The following article demonstrates the importance and need to fully incorporate the various occupational therapy benefits into the catalog of the National Health Fund (FONASA, from now on) considering this profession as a provider of temporary and permanent rehabilitation services in users with different pathologies and / or diseases that last throughout the life cycle. The general aim was "to know the meanings that the relatives of users in need of permanent rehabilitation have with respect to the situation that some professional occupational therapy services or benefits are not included in the FONASA system.

This research is based on the discourses of relatives (Parents-Mothers) of children with cerebral palsy of a group belonging to the Magallanes region, where it is proposed from a qualitative methodology (application of semi-structured interviews).

Results suggest that the relatives of children with cerebral palsy express knowing the role and importance of an occupational therapist in the rehabilitation process of their children, emphasizing that it is necessary for the State to provide the necessary resources, so that the benefits of professionals can be more accessible.

Finally, this article not only attempts to validate the interventions of occupational therapists to incorporate them into the FONASA catalog of benefits, but also rescues the subjective perceptions of those who depend on these interventions, and who are, in some sense, unable to exercise their right to a public health accessible to their needs, from a human rights perspective.

\section{KEYWORDS}

Occupational Therapy, National Health Fund, Public health, Cerebral palsy, Rehabilitation, Human Rights.

Recibido:21/08/2019

Aceptado: 04/05/2021 


\section{INTRODUCCIÓN}

Durante el mes de julio del año 2017, el equipo de investigación (Hoy terapeutas ocupacionales en la Región de Magallanes) realizó una entrevista a M.C. Terapeuta Ocupacional dirigente del Colegio de T.O en Chile, quien fue el encargado del proceso de reestructuración de prestaciones y servicios asistenciales de la profesión en FONASA. ${ }^{5}$

Esta entrevista nos entregó los antecedentes que a continuación se describen. Durante los años 2014-2015 FONASA reformula el catálogo de prestaciones, tras la solicitud del presidente del colegio de terapeutas ocupacionales de Chile, FONASA invita a los profesionales T.O a participar en las mesas de discusión y reformulación de antecedentes. Fueron un total de 18 mesas de discusión y descripción rigurosa las que se constituyeron para explicitar cuales son las prestaciones que deben ser ingresadas al sistema, destacando el tipo de atención, duración, lugar para ser efectuada, copago, entre otros; y los beneficios para los T.O y usuarios.

Se afirma que el 88\% de los T.O activos (colegiados o asociados a organizaciones) se desempeñan en el ámbito privado (Collado, 2015) Resultado un poco paradójico, considerando que cada vez son más los terapeutas que apuestan por la creación de empresas de servicios terapéuticos, priorizando así su autoempleo y oferta laboral para otros colegas, lo que demuestra la falta de reconocimiento de la labor de estos profesionales por parte del estado, que en la actualidad no los deja desempeñarse de forma activa en servicios públicos, desconociendo la necesidad de integrarlos a un equipo multidisciplinario esencial para la promoción, prevención y rehabilitación en salud. Destacando una vulneración de derechos para los usuarios de recibir una atención integral y de calidad por parte de este personal esencial en salud pública. ${ }^{6}$

5 Vale destacar que en el año 2020 se incorporaron prestaciones de Terapia Ocupacional al catalogo FONASA. Véase https://fonasa.cl/ sites/fonasa/noticia/Fonasa incorpora 67 nuevas coberturas

6 Esta información, al momento del estudio, no se encontraba sistematizada en algún documento, todo lo expuesto corresponde a antecedentes proporcionados por Manuel Curihuinca en la entrevista citada.
En base a estos antecedentes, un grupo de alumnas de la carrera de T.O de la Universidad de Magallanes, deciden realizar su seminario de investigación en base a la necesidad expresada y evidenciada en algunos de los familiares de usuarios por acceder a estas prestaciones con un arancel accesible, pretendiendo dilucidar algunos efectos de la exclusión parcial de la T.O en FONASA, incorporando las experiencias y significados de las familias y usuarios sobre el quehacer de los T.O, en niños/as con diagnóstico de PC. ${ }^{7}$

Justificación del problema: Referirse a la situación que la T.O no está dentro de fONASA es algo impreciso. Si se cubren prestaciones del área, éstas están condicionadas desde dos modalidades: la primera, por la que se rigen estas prestaciones es el Catálogo Modalidad Asociada a la Institución (MAI) y la segunda, es el Catálogo Modalidad Libre Elección (MDE); desde esta perspectiva es importante mencionar que las prestaciones de la T.O solo se rigen sobre el catálogo MAl, entonces, no está considerada en la modalidad libre elección (MLE), lo que significa que esta no es accesible para toda la población. (Olivares, et.al, 2015).

Formar parte de la modalidad MAI, permite que las prestaciones a T.O se dirijan desde la atención única y exclusivamente pública, permitiendo así que los ejercicios de sus intervenciones solo puedan ser requeridas por instituciones que tengan al profesional asociado a FONASA Como por ejemplo un hospital; exceptuando así a toda persona natural que requiera de los servicios asistenciales de un terapeuta ocupacional, lo que perjudica a una amplia gama de población de usuarios que requieren de atenciones de Terapia ocupacional. (Gajardo, et.al, 2017).

Un grupo en particular que se ve afectado por esta arbitraria exclusión, son aquellos que requieren de tratamientos e intervenciones de rehabilitación permanente. Nosotros tomamos contacto con ACRESU (Agrupación Creciendo por un Sueño) que aglutina a padres, madres y otros familiares de usuarios con PC, principalmente usuarios pediátricos entre o y 18 años.

\footnotetext{
7 Es importante destacar que al momento de la realización del estudio (2017-2018) aún no se hacía efectiva la incorporación de la T.O a nuevas modalidades de prestaciones en FONASA.
} 
Se decidió trabajar con familiares de ,usuarios con diagnóstico de PC en la etapa de la infancia debido a la necesidad significativa y permanente que poseen estos niños/as y sus familias de recibir prestaciones, tratamientos de rehabilitación y ayudas técnicas, necesarios para satisfacer necesidades y mejorar su calidad de vida.

\section{"La Parálisis Cerebral Infantil define a un grupo de trastornos motores, caracterizados por una alte- ración en el control de los movimientos y la pos- tura, causados por una lesión cerebral ocurrida en el período de crecimiento acelerado del cere- bro (desde el desarrollo prenatal hasta los cinco años de vida). Si bien la lesión es no progresiva, las manifestaciones clínicas pueden variar con el tiempo. Constituye en la actualidad la princi- pal causa de discapacidad física en la infancia (Weitzman, 2005, p. 46).}

El impacto ocupacional de quienes son diagnosticados con este trastorno neuromotor es de gran envergadura, no solo para quien lo experimenta, sino también para su familia y entorno, debido a que se presentan dificultades considerables en la independencia de la persona en las diversas áreas de ocupación (López, 2018). Enfocándose en pediátricos de o a 18 años, las áreas mayormente afectadas corresponden a: actividades de la vida diaria básica e instrumental, educación, juego y participación social. Es por aquello que los/as niños/as y adolescentes requieren de un tratamiento continuo para favorecer y/o facilitar su desempeño ocupacional y por sobre todo su participación e inclusión social.

Para una rehabilitación de calidad y duradera en el tiempo, se requiere de una extensa lista de profesionales de la salud, interesados en mejorar la calidad de vida del usuario y su familia (neurólogo, fisiatra, traumatólogo, ortopedista, kinesiólogo, terapeuta ocupacional, fonoaudiólogo, etc.). (Vilaboy Filgueiras y Vásquez Vásquez, 2011).

En el caso de la Agrupación Creciendo por un Sueño, los padres y/o cuidadores de usuarios con necesidades de rehabilitación permanente, han logrado acceder, en su mayoría, a las prestaciones que el Centro de Rehabilitación de la ciudad de Punta Arenas les ofrece como opciones de intervención. Los testimonios citados en la investigación destacan la limitación y acotación que tienen las sesiones de la profesión, demostrando la necesidad de recibir una atención de manera continua y permanente.

En base a estos antecedentes, este estudio plantea las siguientes preguntas y objetivos de investigación: ¿Cuáles son los significados que le otorgan usuarios con necesidad de rehabilitación permanente, respecto a la situación de que algunos servicios o prestaciones profesionales asistenciales de T.O no se encuentren incluidos en el sistema FONASA?

Objetivo general: Conocer los significados que tienen los usuarios con necesidad de rehabilitación permanente y sus familias, respecto a la situación de que algunos servicios o prestaciones profesionales asistenciales de T.O no se encuentren incluidos en el sistema FONASA, en el caso de la Agrupación Creciendo por un Sueño.

Objetivos específicos: Identificar los niveles de conocimiento que tienen los usuarios y familiares sobre la labor del T.O en el proceso de rehabilitación. Identificar las dificultades y limitaciones económicas y de acceso a prestación que implica para una familia de usuarios con necesidad de rehabilitación permanente. Conocer las expectativas (valoración y necesidades) que tienen los usuarios respecto a la incorporación de las prestaciones de T.O En FONASA.

\section{MARCO TEÓRICO CONCEPTUAL}

Hacia un concepto multidimensional de salud: disensos científicos a propósito de la salud como un constructo polisémico. La Organización Mundial de la Salud estableció como definición de salud el "estado de completo bienestar físico, mental y social” (OMS, 2013). A esta definición de salud se han ido incorporando otras dimensiones, como la capacidad de funcionar o la salud como un fenómeno continuo y dinámico a lo largo del tiempo, hasta llegar a instaurarse la idea de que la salud es un fenómeno multidimensional. Sin embargo, debemos escapar del idealismo ingenuo que supone que la salud y las decisiones políticas que giran en torno a ella carecen de neutralidad, al contrario, siempre son arbitrarias e ideológicas.

Es importante conceptualizar lo que se entiende como salud pública: 


\begin{abstract}
"La Salud Pública tiene una larga historia que se inicia con labores de gobiernos al cuidado de problemáticas individuales o puntuales evolucionando a lo que es hoy, una Salud Pública más contemporánea, preocupada por los grupos o colectivos humanos y su incidencia en el desarrollo de mejores condiciones de vida para la población, focalizando en grupos vulnerables, que a su vez exigen sus derechos y están atentos a las redes que el propio sistema otorga". (ArayaVallespir, 2012, p. 57).
\end{abstract}

El concepto de salud pública, a pesar de su hegemonía y tradición, no es un concepto exclusivo o la única forma de entender la salud desde las responsabilidades del Estado y la sociedad, sino que también existen otras alternativas, de las cuales han nacido otras posibilidades de entender la salud desde lo público-estatal como por ejemplo la salud colectiva, la cual tiene sus orígenes en Brasil y Ecuador y se ha expandido rápidamente en otros países de Latinoamérica. (Casallas-Murillo, 2017; Breilh, 2013).

La Salud Colectiva se define como: un conjunto complejo de saberes y prácticas, técnicas científicas y culturales, ideológicas, políticas y económicas, relacionados al campo de la salud, involucrando desde las organizaciones que prestan "asistencia" a la salud de las poblaciones hasta las instituciones de enseñanza, de investigación yorganizaciones de la sociedad civil (osc) que puedan tener un carácter científico o sindical interesadas en defender el sistema público de salud en el marco de la lucha por el "derecho a la salud" de la ciudadanía (Liborio, 2013, p. 139) De esta forma la Salud Colectiva se consolida como campo científico y ámbito de prácticas abierto a la incorporación de propuestas innovadoras.

La importancia está dada, una vez más, en el reconocimiento de que el usuario es protagonista del proceso, no como un receptor de lo que se le otorga desde la visión arcaica de salud y enfermedad, sino como un agente activo de conocimiento, capaz de significar y aportar creativa y activamente a sus experiencias en salud (Liborio, 2013).

Igualmente, se asume que las desigualdades en salud constituyen una prioridad creciente, en la medida que éstas se han hecho más evidentes y las estrategias implementadas, han fracasado (Pizarro, 2005). La distribución desigual de poder, bienes, servicios e ingresos, combinados con el insuficiente resultado de la gestión política y programas sociales deficientes, afectan las condiciones de vida y específicamente, determinan las condiciones en que las personas nacen, crecen, viven, trabajan y envejecen (Muñoz, 2014)

Desde esta perspectiva, estamos ante un Estado débil en temas de cobertura universal, de accesibilidad y de prestaciones equitativas para los usuarios. Son amplios y extensos los sectores de la población que no pueden acceder a salud de calidad, desde este criterio podemos inferir que la salud colectiva propicia una forma de representar y concebir los procesos de salud/enfermedad como una construcción con actores diversos, no obstante, aquellas personas que ven limitadas sus posibilidades de acceso a la atención pública, ven restringidas sus opciones de participar en estos procesos, y la exclusión de una disciplina como la T.O viene a instalarse como un indicador de limitación que afecta a la ciudadanía en general. (Tamayo, et.al, 2018; Padrón y Roman, 2010)

\section{TERAPIA OCUPACIONAL E IGUALDAD EN EL ACCESO A LA SALUD}

Diversos terapeutas ocupacionales han discutido con respecto a los constreñimientos éticos y políticos que implica asumir que sus prestaciones e intervenciones están condicionadas por decisiones políticas (y sus inmanentes cargas ideológicas), por contextos económicos y sociales y por significados subjetivos que irrumpen desde la cultura y cotidianidad situada de sus sujetos de intervención. (Espinoza, 2016).

Además, desde la visión de la T.O, en que la ocupación es el medio o el fin, a través del cual los seres humanos mantenemos salud y bienestar (Kielhofner, 2003), debiese resultarnos un imperativo ético pensar sobre la realidad actual de la provisión de servicios de salud en el país; los obstáculos y posibilidades que ha brindado la protocolización de intervenciones para la esencia y principios fundantes de la disciplina y la práctica centrada en el cliente; la forma de priorización para la atención que se establece para los usuarios, las patologías GES y nO GES; entendidas como "garantías de acceso, calidad, protección financiera y oportunidad con que deben ser otorgadas las prestaciones asociadas a un conjunto priorizado de programas, enfermedades o condiciones de salud 
que señale el decreto correspondiente". (Superintendencia, 2020), las lógicas de funcionamiento del sistema privado en salud, principalmente referido a altos costos y la discriminación que imponen los mismos; el acceso universal de prestaciones de la disciplina (y nuestra reiterada necesidad de entrar en modalidad libre elección de FONASA)" (Muñoz, 2014) Agrega la autora; Requerimos, por tanto, de profesionales terapeutas ocupacionales capaces de pensar sobre la contingencia social, de entender cómo la globalización, la sustentabilidad del planeta, la pobreza y la marginación; las formas de producción, las crisis económicas, las fuentes y condiciones de (des)empleo, los desastres naturales, las violaciones a los derechos humanos, y de las libertades individuales, afectan una forma colectiva e individual de enfrentarnos a la vida. "Una sociedad que gesta inequidad nunca será capaz de superarla, por mucha y buena política social que cree; la solución está en generar sujetos sociales con potencial y gusto por la transformación social, que en sus procesos de trabajo y mediante apropiación de poder - democráticamente responsable-, permeen los macro sectores sociales desde aquello que acontece en un box de atención, en una sala de clases, de rehabilitación, o en un taller vecinal"(Muñoz, 2014, p. 79).

Finalmente, Emeric (2015) en sintonía con Muñoz (2014) se pregunta "Acaso la T.O no basa parte de sus pilares filosóficos esenciales en posicionamientos ideológicos tales como: "todas las personas son iguales en derechos", "todas las personas merecen gozar de oportunidades para disfrutar de una vida digna". ¿No se traducen (o debería), a la vez, estos posicionamientos ideológicos, en propuestas políticas concretas y tangibles en nuestra vida cotidiana? ¿En leyes, decretos, planes, programas... que posibilitan que los/as Terapeutas Ocupacionales nos aproximemos al cumplimiento de tales fines mientras desempeñamos nuestra actividad profesional?

Eventualmente estas interrogantes son más potentes cuando la/él profesional interactúa con realidades caracterizadas por la vulnerabilidad, la precariedad, el apartheid ocupacional y otros (Kronenberg, 2007).

En este sentido es importante enmarcar las experiencias subjetivas de los usuarios en salud dentro de un contexto de determinantes sociales que condicionan las oportunidades de los usuarios, y porque no decirlo, la vida misma de las personas. La exclusión parcial de la T.O en FONASA constituye una exclusión arbitraria que deja a un espectro importante de la población en Chile sin acceso a las prestaciones de estos profesionales, que, al mismo tiempo, son "empujados" a tener que ejercer en centros de atención privada donde los costos particulares son elevados y muchas veces inaccesibles para un amplio número de la población. En función de lo anterior Merino (2016) refiriéndose a las etapas de democratización de la salud pública en la historia de Chile, enfatiza en que el Estado debe garantizar la salud como un derecho social y humano para todas las personas.

\section{MÉTOdo}

Esta investigación es de tipo descriptivo-exploratorio y se abordó a partir de una metodología cualitativa (Baeza, 2002). Se aplicaron 15 entrevistas semi-estructuradas a familiares y cuidadores de usuarios pediátricos con PC, de la Agrupación Creciendo por un Sueño, estos participantes fueron elegidos a través de un muestreo teórico, debido a que los miembros de la misma responden a un colectivo de estudio susceptible de proporcionarnos la información pertinente para dar respuesta a nuestra pregunta de investigación y objetivos de estudio. Los datos textuales fueron transcritos literalmente en documentos Word, para posteriormente ser tratados en el Software Atlas ti versión 7 (Free trial). Se aplicó un proceso de codificación (abierta y axial) donde encontraremos segmentos representativos de códigos emergentes y de códigos teóricos (extraídos de una revisión de literatura) para posteriormente reducir nuestra cantidad de códigos a través de la operación de "agrupación de familia de códigos" lo que nos condujo a crear diagramas o mapas de relaciones entre los códigos (Strauss y Corbin, 2002).

Es importante destacar que esta investigación fue aprobada por el "Comité de ética científico" de la Universidad de Magallanes. Esta evaluación garantiza que los aspectos éticos, los derechos y dignidad de los participantes fueron adecuadamente resguardados.

\section{Resultados}

A continuación, se exponen los resultados a través de categorías obtenidas por medio de las entrevistas semi estructuradas realizadas. 


\section{Conocimiento sobre la Terapia ocupacional}

Con respecto al conocimiento que tienen los participantes sobre la labor y las intervenciones que realiza un T.O.

Según los relatos de los entrevistados, las personas logran tener una visión según sus propias experiencias y las atenciones recibidas por sus hijos y lo que el TO ha trabajado con ellos, especificando mayormente el trabajo con niños, sin embargo, logran identificar que se puede trabajar con diversos grupos etarios y en otras áreas de forma más generalizada.

"La parte como de hospitales, por ejemplo, también pueden estar en el tema de salud mental, Psiquiátrico, en poli también, pueden estar en... en educación también pueden desenvolverse ahí, también pueden estar en el tema, a ver cuál me falta, en niños en la geriatría..." (Fragmento de entrevista de investigación. P4)

"Ellos trabajan no solamente con niños, sino que también con adolescentes, con adultos en distintas áreas $\mathrm{mmmm}$ para distintos tipos de condiciones $\mathrm{mmmmm}$ déficit atencional o terapia con retos múltiples..." (Fragmento de entrevista de investigación. $P_{3}$ ).

\subsection{INTERVENCIÓN DE TERAPIA OCUPACIONAL}

De los elementos que contienen los T.O se concentra en las áreas de desempeño funcional, prestando esta área el principal reconocimiento de su labor.

"Lo derivaron directamente con Terapia Ocupacional y alli ha trabajado el agarre, por ejemplo, a través del juego, emm cómo seguir instrucciones, ese tipo de cosas que ha ido como avanzando, evolucionando..." (Fragmento de entrevista de investigación. P1).

"Posicionamientos, ayuda con, ay! Como se llama esto... ayudas técnicas y estimulación sensorial a través de olores, a través de colores, de texturas, la estimulación a través del movimiento también..." (Fragmento de entrevista de investigación. P8).

\section{2-BENEFICIOS Y VALORACIÓN DE LAS INTERVENCIONES DE T.O.}

Según el análisis de los resultados, el beneficio que aporta la atención de T.O tiene un impacto diverso, en las realidades de los participantes de la investigación, principalmente en la mejoría de la calidad de vida por medio de la intervención de dicha disciplina. Además, es relevante que los participantes apunten al concepto de "calidad de vida". Si bien la salud es un elemento ineludible de la misma, en ningún caso la determina, por lo tanto los usuarios, identifican en la labor de los T.O como algo más que un prestador de servicios clínicos asistenciales para recuperar funcionalidad.

"Es que la terapia ocupacional em por ejemplo, cuando la gente recién conoce terapia ocupacional sabe qué, que lo puede ayudar en un montón de ámbitos, entonces pienso que lo beneficios iban a ser en mejorar la calidad de vida..." (Fragmento entrevista de investigación, p. 5).

Los participantes mencionan principalmente el desconocimiento que existe por el rol de los T.O, esclareciendo que ellos conocen ciertas acciones debido a que sus hijos han recibido sus intervenciones.

"Si yo creo que el trabajo de la terapia ocupacional abarca otros aspectos de la rehabilitación, que no tienen que ver solamente con la parte física, digámoslo así, Siento que en términos generales la comunidad no conoce mucho la labor del terapeuta y yo creo que es tan importante como un kine, o sea son la otra parte de la rehabilitación, el tema de la rehabilitación, toda esa parte digamos se complementan ambas disciplinas...". 
FIGURA 1 SOBRE EL CONOCIMIENTO DE LOS USUAROS CON RESPECTO A LAS INTERVENCIONES EN TERAPIA OCUPACIONAL (ELABORACIÓN PROPIA).

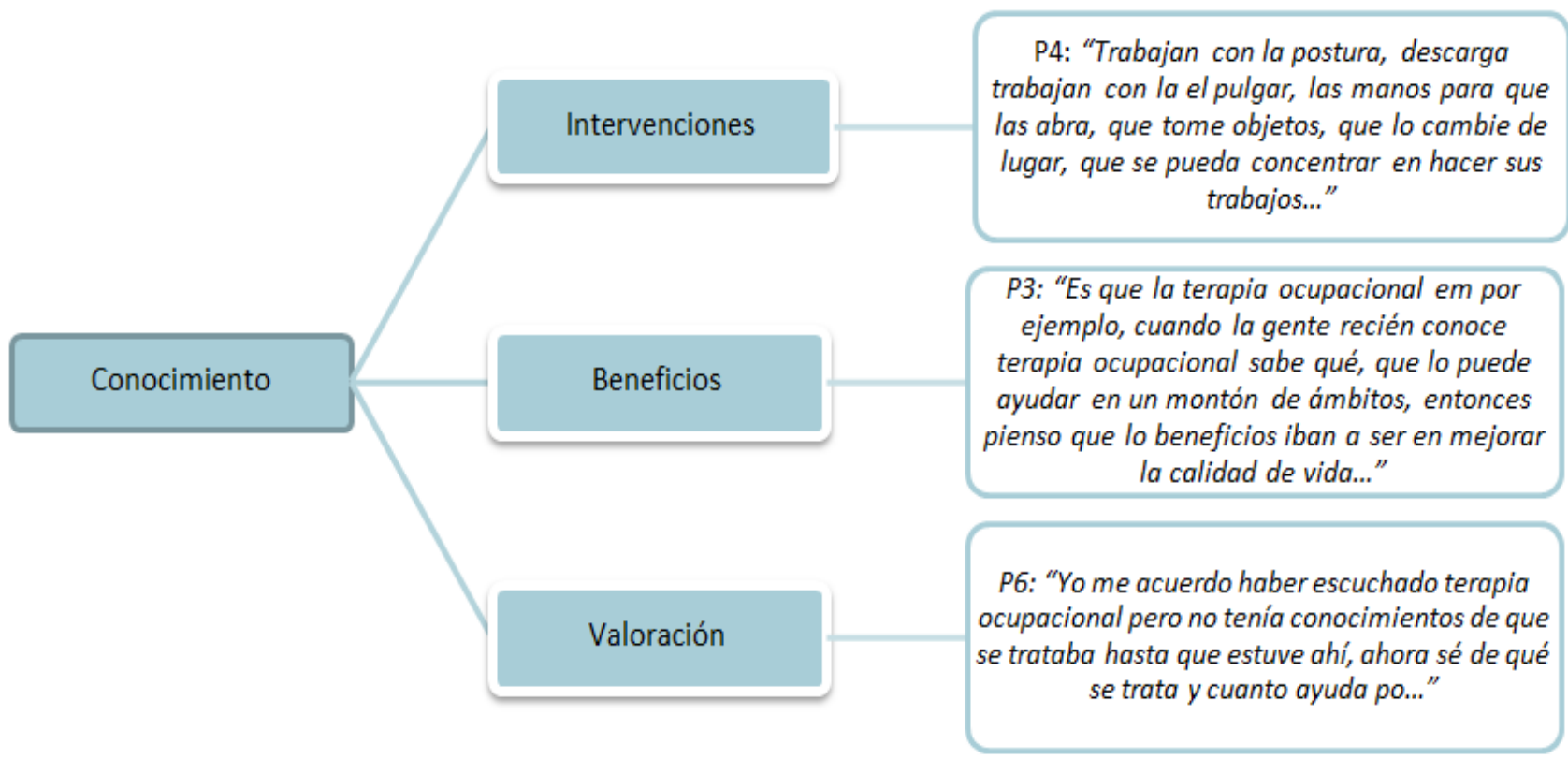

(Basada en los discursos de los participantes)

\section{Sesiones temporalmente limitadas de Terapia Ocupacional}

Los testimonios citados destacan la limitación y acotación que tienen las sesiones de la profesión en diversas entidades, demostrando la necesidad que poseen sus hijos por recibir esta atención de manera continua y permanente en el tiempo, para poder mejorar la calidad de vida de aquellos.

"A mí me pasa, es que tiene, terapia una vez a la semana media hora entonces es como mmmm se me hace poco realmente eeee siento que a otros profesionales le han sacado más provecho alX..." (Fragmento de entrevista de investigación p6)

Yo creo que es corta, ambas, porque piensa que es media hora, mientras uno llega, saca la ropa, perdiste 5 minutos después mientras comienza, igual en el fondo yo creo que es como 20 minutos integrales así enfocados en la atención, igual yo creo que es poco, yo creo que debería ser el doble, no sé, unos 40 o 45 minutos..." (Fragmento de entrevista de investigación p11).

"Hay mucha gente que se queda sin prestaciones en el centro y necesita todavía de un kine de un terapeuta de un equipo..." (Fragmento de entrevista de investigación p1)

\subsection{Costos DE INTERVENCIONES}

Los resultados evidenciados en los discursos declarados señalan que la mayoría de las atenciones particulares realizadas por un T.O rodean el valor de treinta mil pesos (según las experiencias de los entrevistado), haciéndolas en variadas ocasiones poco accesibles para la población de clase media a baja y sin una inspección que apele a la seguridad de una intervención de calidad.

Podemos destacar que existe un valor aproximado y en algunos casos certero a lo que cobra un profesional de forma particular, este cobro no posee ninguna regularización y se encuentra establecido en base a los principios y/o ética de cada profesional. 
"Si, si y están caras si uno eh si el cobro particular séque están entre 30.000, 35.00o y es la sesión o la evaluación entonces obviamente si yo les hablo de 10 sesiones a una persona estamos hablando por parte baja trecientas lucas que van a hacer em... en dos meses, dos meses y medio entonces obviamente es plata po y la gente obviamente va a elegir em... no utilizar la terapia ocupacional, aunque sepan que es algo que les va a servir..." (Fragmento de entrevista de investigación p1)

"Creo que es como una sesión algo así son como 20 o zomil pesos media hora..." (Fragmento de entrevista de investigación p7).

\subsection{FINANCIAMIENTO DE AYUDAS TÉCNICAS}

Las "ayudas técnicas (en adelante A.T.) son productos externos fabricado especialmente o ampliamente disponibles, cuya principal finalidad es mantener o mejorar la independencia yel funcionamiento de las personas y, por tanto promover su bienestar" (Minsal, 2017) El financiamiento de las AT, alude al constante requerimiento en usuarios pediátricos, las cuales son necesarias, debido a que se encuentran en edad de desarrollo, siendo aquellas ineludibles para la rehabilitación de sus hijos. Algunos relatos, hacen énfasis en el compromiso y ayuda que reciben desde el departamento de T.O de la Universidad de Magallanes, declarando que, desde el servicio de profesionales y alumnos de la carrera, acceden a A.T de bajo costo para quienes la fabrican, las cuales son otorgadas a ellos sin costo alguno, gracias a la confección y distribución de las mismas en la casa funcional de TO. De lo antepuesto, destacan los siguientes discursos:

$\mathrm{P}_{1}$ : "Entonces te piden una a los dos años otra a los cuatro y te piden una a los seis, ya y entonces emmmm cortaron eso refinanciaron dijeron ya tal persona se tienen que hacer cargo esto, estas partes del gobierno se tienen que hacer cargo, pero nunca le llegaron las platas a ninguna de las partes..."

P4: "entonces para las ayudas técnicas es muy muy complicado el sistema y mal hecho porque se supone que de o a 7 años es una edad critica, o sea donde más ayudas técnicas necesitan..."

Lo que refieren los participantes de la investigación en esta subcategoría se relaciona con las limitaciones del sistema público respecto a las A.T ya que no existe institución que financie aquellas, para menores de 7 años, siendo que los pediátricos están en una etapa crítica de desarrollo donde estas herramientas son fundamentales para su desarrollo.

Según lo recopilado, el financiamiento de las A.T en nuestro país es un tema complejo, debido a que existen escasas instituciones que entreguen de manera accesibles estas herramientas, siendo costeadas principalmente de manera particular y en algunos casos, por medio del centro de rehabilitación de la ciudad y/o Servicio Nacional de la Discapacidad, ambas instituciones mencionadas con anterioridad no confirman la obtención del producto tras la postulación, ni aseguran que lleguen a la brevedad, pudiendo demorar años la entrega.

\subsection{LIMITACIONES DEL SISTEMA PÚBLICO RESPECTO A LAS AYUDAS TÉCNICAS}

Según los resultados expuestos en los discursos analizados la incorporación de las prestaciones de servicio de la T.O en FONASA sería un beneficio para todas las personas que requieran de la atención de esta profesión teniendo un mejor y mayor alcance tanto en factores económicos como sociales dentro de la población Chilena, además existiría una regularización con respecto a la calidad de las atenciones que prestan los T.O de forma particular o dentro de las instituciones que utilicen este tipo de previsión

Este ítem alude principalmente a la necesidad de que se creen instituciones que abarquen a todos los rangos etarios y estratos sociales para poder adquirir una A.T de calidad y a un precio accesible a los bolsillos de los chilenos, además de destacar que los catálogos de las entidades actuales del país que entregan estas ayudas poseen A.T arcaicas, limitando la posibilidad de mejorar y adquirir sofisticadas y/o avanzadas.

"No, algunas cosas solos con ayuda igual de nuestros amigos, familia y todo, pero generalmente del bolsillo, y otras también, algunas ayudas vinieron de la universidad de bajo costo a través 
FIGURA 2 SOBRE LAS DIFICULTADES Y LIMITACIONES PARA ACCEDER A PRESTACIONES DE T.O (ELABORACIÓN PROPIA):

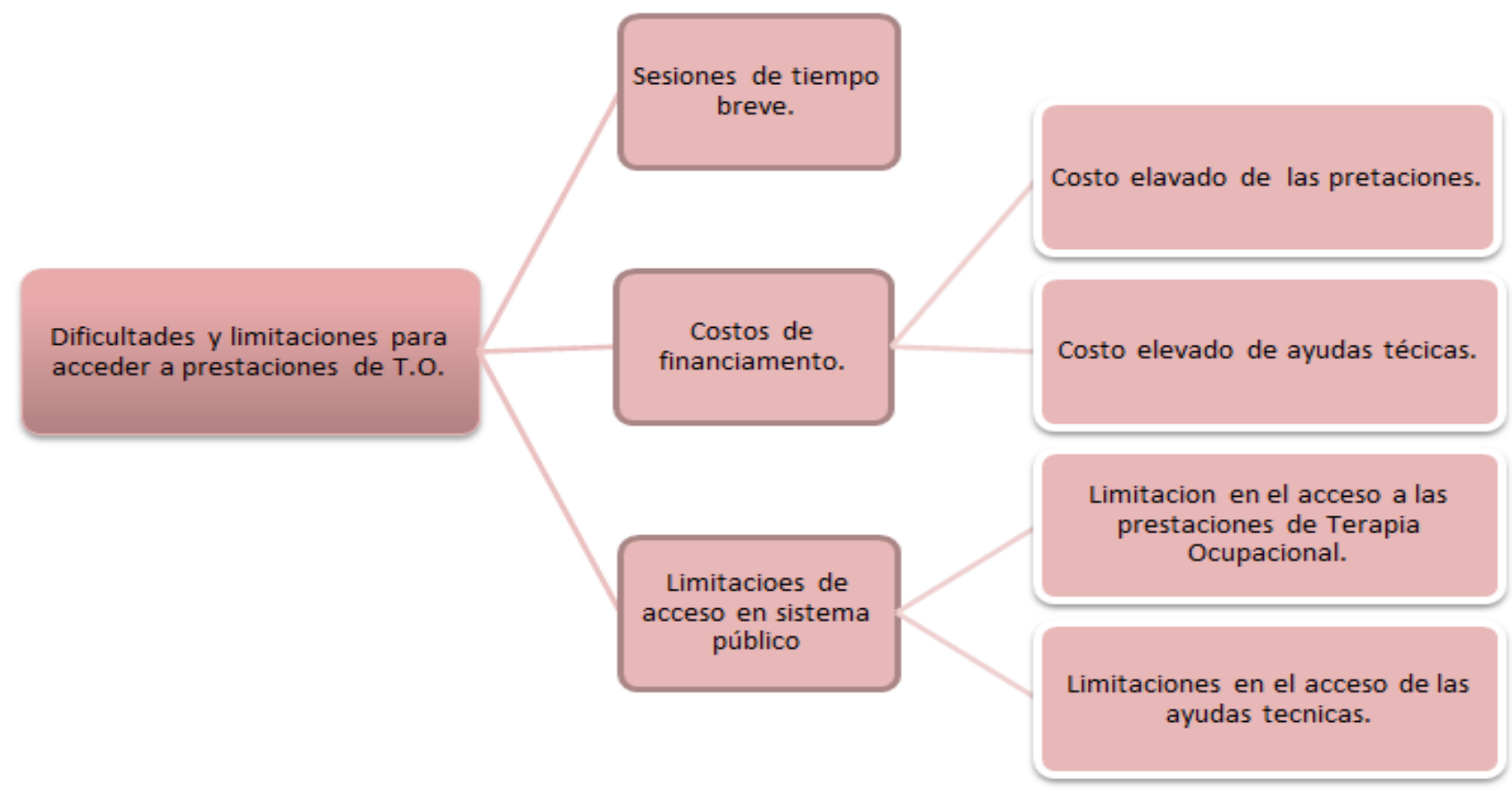

(Basada en los discursos de los participantes)

de un programa, que fue una bañera que nos sirvió mucho, pero después llego la bañera oficial de SENADIS Ya..." (Fragmento entrevista de investigación p5)

"Uy! Un montón, por decirte el uPSI sale quinientos mil pesos, el bipedestador sale como quinientos, el siting sale como trescientos, las órtesis del centro habremos gastado unas doscientas más, la silla neurológica no la compramos nosotros, la donaron..." (Fragmento de entrevista de investigación p7)

\section{Expectativas y necesidades sobre la incorporación definitiva de la T.O al sistema FONASA}

Principalmente los participantes destacan la validez de la profesión como necesaria en el ámbito de la salud y cómo su incorporación al sistema público favorecería tanto a sus hijos como a otros usuarios que requieran de las intervenciones de T.O y el beneficio de la accesibilidad a aquellas prestaciones.

"Si lo que quería decir, voy al punto que yo creo, no cuesta nada dar las prestaciones, total la persona, el usuario va a decidir si va o no, si toma las prestaciones, o sea si existen las prestaciones de Terapia Ocupacional, nosotros que sabemos es importante, vamos a ir y lo vamos a tomar, pero otras personas que no lo conocen, no lo van a tomar. O sea... Es importante que este la oferta, que se dé la oportunidad de tomarlo..." (Fragmento de entrevista de investigación p12).

"Poder acceder a por ejemplo para mi es difícil llevar siempre a X a terapia al centro, yo tengo que pagar taxi ida y vuelta y no es solo una terapia si no que ella necesita más terapia, e tal vez si un terapeuta estuviera por FONASA podría pagarlo 


\section{FIGURA 3 SOBRE LAS EXPECTATIVAS CON RESPECTO A LA INCORPORACION DEFINITIVA DE LA T.O EN FONASA (ELABORACIÓN PROPIA)}

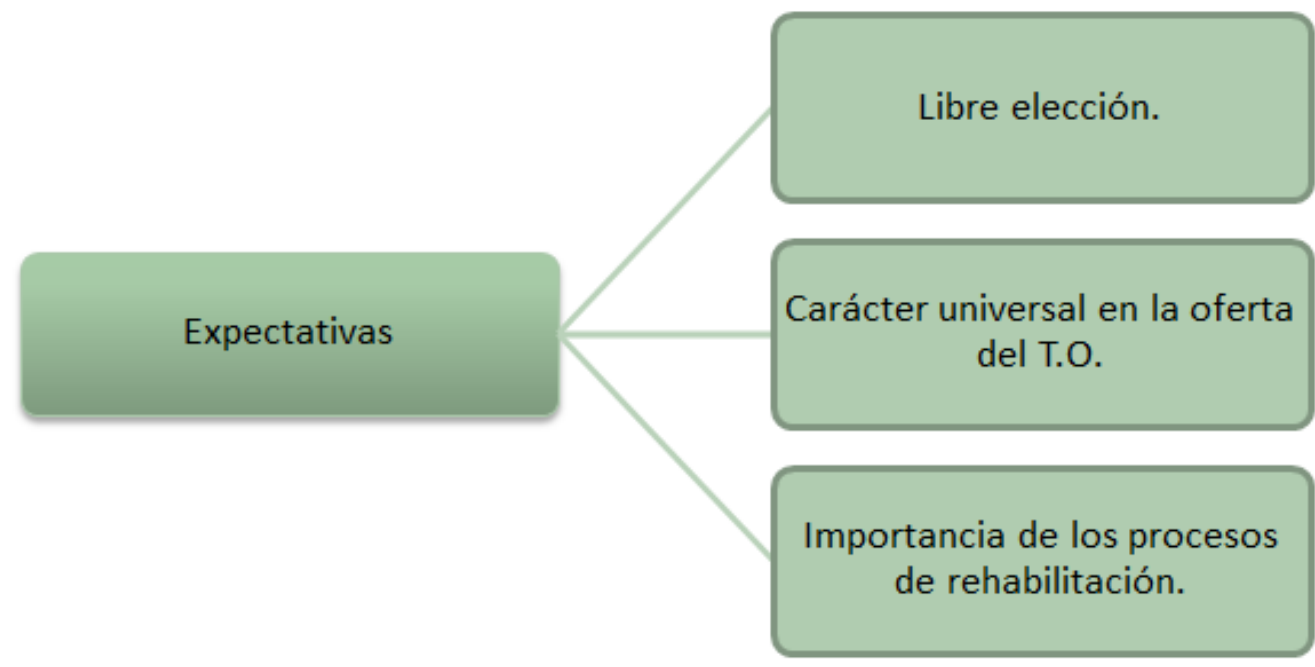

(Basada en los discursos de los participantes)

un costo menor o esas mismas ayudas técnicas si de alguna manera se puede que una parte la financie el estado y que uno la pueda agilizar más rápido que pueda ser más rápido el beneficio..." (Fragmento de entrevista de investigación p3).

\section{Discusión}

Según los resultados obtenidos en la investigación, nos encontramos en un primer momento con las aristas de "valoración y reconocimiento" del trabajo y los aportes de las intervenciones de T.O. en los usuarios con P.C. Las personas que han adquirido atención de estos profesionales reconocen solo aquellas áreas de trabajo en las que han sido intervenidos, enunciando desconocimiento de la labor en otras. Sin embargo, existe claridad del aporte que esta profesión otorga en la calidad de vida de las personas que requieran de la atención de un Terapeuta Ocupacional y cómo esta disciplina otorga un valor significativo en el estado de salud de sus hijos. Parcialmente, podemos decir que la gente al reconocer la importancia de los servicios ofrecidos por la T.O validan a la profesión. (Olivares, et.al, 2015).

El debate sobre la validación de esta disciplina, como una ciencia de la salud es problemático, debido a que su origen epistemológico es la ciencia de la ocupación, aunque muchas veces se erige como una disciplina interdisciplinaria que dialoga con otros saberes, por ejemplo, de las ciencias sociales (Solsona, 2018). En esta misma línea, Paredes, Vásquez y Muñoz (2014), destacan el intento de posicionar conocimientos vinculados al impacto que tiene la ocupación en la salud y en la calidad de vida de las personas, así como también destacan la dificultad existente de conceptualizar la identidad de la disciplina, como producto de las diferentes corrientes ideológicas y epistemológicas que potencian el hacer de los T.O. Sin embargo, a pesar de la constante lucha porque se reconozca la labor de la disciplina, sigue siendo desconocida para muchos, dificultando así su trayectoria y pleno desempeño de sus profesionales, limitando a la población al acceso de aquella. 
Por otro lado, un porcentaje significativo de la población entrevistada estima que la incorporación de las prestaciones de T.O a FONASA beneficiaria de manera positiva a la población, la cual podría optar a servicios de manera permanente en el sector público, evitando la atención particular y los costos elevados que estas poseen, al mismo tiempo de reconocer que esto favoreceria también el hecho de que los costos serian regularizados y se garantizaría la labor de un profesional capacitado, además de brindar accesibilidad de atención en los diferentes estratos sociales, aumentando la demanda de usuarios que puedan acceder a la intervención y por consiguiente, favorecería la atención oportuna y la óptima rehabilitación de quienes requieran de su atención.

La no incorporación total de la T.O a FONASA por parte del Estado, priva parcialmente a las personas de acceder a un derecho de atención universal, y atenta contra el horizonte normativo de la definición de salud entregado por la OMS (2013) con respecto al objetivo de la consecución de un bienestar integral, físico, emocional y social en las personas.

Otra arista importante encontrada en la investigación, refiere a que las personas, generalmente deben gestionar los procesos de rehabilitación de sus familiares con P.C., de forma particular, desplegando diversos recursos para acceder a terapias. Esto viene a evidenciar un parcial abandono del Estado, cuyos vacíos deben ser subsanados por intensas gestiones familiares, muchas veces gastando lo que no se tiene y recurriendo a fundaciones privadas que ofrecen los servicios a menor costo. Entonces, la exclusión de una disciplina como la T.O en el sistema público de salud, viene a instalarse como un indicador de limitación que afecta a la ciudadanía en general (Tamayo, et.al, 2018; Padrón y Roman, 2010).

Otra lectura que puede hacerse de la exclusión de algunas prestaciones de T.O en FONASA, tiene que ver con el "Apartheid ocupacional". Kronenberg (2007) afirma que este se relaciona con limitaciones políticas que afectan todos los aspectos de la vida cotidiana, incluyendo a la salud, y que se explicar principalmente por la pobreza crónica y la desigualdad. Bajo esta premisa, podemos inferir que siempre serán los pobres, o aquellos grupos mas vulnerables los que serán susceptibles de apartheid, sobre todo considerando que su baja cantidad de activos financieros y recursos, les impide "elegir" opciones de tratamientos de rehabilitación de forma particular, por el alto costo de los mismos.

En definitiva, el Estado debe ser garante no solamente del acceso universal a este tipo de prestaciones, sino, además, de considerar las necesidades especificas de los grupos humanos (Muñoz, 2014). En nuestro caso de estudio, para los familiares y usuarios con P.C., como la literatura consultada ha evidenciado (López, 2018; Weitzman, 2005), la recuperación o logro de autonomía, independencia y funcionalidad como parámetros de bienestar humano, subyacen a las expectativas de acceso universal a prestaciones de T.O en el sistema publico de salud. No tiene que ver solamente con una rehabilitación desde el punto de visto físico-médico, sino que se relaciona con la obtención de un reconocimiento como ciudadanos por parte del Estado, el cual debe garantizar el acceso a la salud como un derecho humano (Merino, 2016).

\section{Conclusión}

El concepto macro dentro de la investigación y que causa mayor relevancia es la rehabilitación permanente como principal necesidad y la limitación en el acceso a ciertas prestaciones necesarias para mejorar la calidad de vida de sus hijos/as, además de los costos elevados que conlleva este tipo de intervención en sesiones particulares. Identificando como foco principal, las necesidades de cada niño perteneciente a la agrupación, con esto hacemos referencia también a las A.T., su alto valor, la necesidad constante de solicitarlas y como se ve limitada la posibilidad de acceder de manera gratuita o a bajo costo de estas asistencias, las cuales serían en directo beneficio de los/las niños/as y jóvenes pertenecientes a la Agrupación Creciendo por un sueño, demostrando un errado enfoque y financiamiento por parte del Estado, donde los pediátricos hasta 7 años no tienen posibilidad estatal de contrarrestar los costos de estas ayudas, teniendo que obtenerlas de forma particular.

Igualmente, se destaca la necesidad en común que tienen los participantes de la investigación en cuanto al requerimiento de una atención integral, permanente y de un costo que se ajuste a la realidad económica regional, familias que representan a los sectores con mayores desventajas socio-económicas, sobre todo para quienes 
se encuentran en una etapa de crecimiento y donde la adquisición de habilidades y destrezas se encuentra en un estado crítico, destacándose así la escasa o nula participación del estado limitando el derecho a la salud, es paradójico pensar que el gobierno entrega diversos beneficios, incluso ha creado políticas públicas a favor de la entrega de servicios y productos gratuitos a la polación de edad adulta y envejecida, minimizando las necesidades actuales de los niños que en plena etapa de crecimiento ven limitado el acceso a una atención y servicio de calidad y accesible económicamente, desigualdad que demuestra que en Chile la salud aun no es un derecho universal.

Finalmente, cuando hablamos de incluir a la T.O en FONASA, lo que se solicita es que esto sea un beneficio para toda la población chilena en calidad de ciudadanos, es decir garantizar un derecho humano para todas las personas y en todos sus rangos etarios.

\section{Bibliografía}

Araya-Vallespir, C. (2013). Salud pública y salud colectiva. Journal Of Oral Research, 2(2), 57-58. https://doi.org/10.17126/joralres.2013.012

Baeza, M. (2002). "De las metodologías cualitativas en investigación científico-social", diseño y uso de instrumentos en la producción de sentido. Concepción: Universidad de Concepción, Chile.

Breilh, J. (2013). La determinación social de la salud como herramienta de transformación hacia una nueva salud pública (salud colectiva). Revista Facultad Nacional de Salud Pública, 31(Suppl. 1), 13-27. Retrieved February 10, 2021, from http://www.scielo.org.co/ scielo.php?script=sci_arttext\&pid=S0120-386X201300040000 2\&lng=en\&tlng=es.

Casallas Murillo, A. L. (2017). La medicina social-salud colectiva latinoamericanas: una visión integradora frente a la salud pública tradicional. Revista Ciencias de la Salud [en línea]. 2017, 15 (3), 397-408 [fecha de Consulta 10 de Febrero de 2021]. ISSN: 1692-7273. Disponible en: https://www.redalyc.org/articulo. oa?id=56253119009.

Collado, A. L. (2015). Terapia ocupacional y economía de mercado ¿Valemos lo que queremos cobrar?: Ocupando los Márgenes. Recuperado de: http://ocupandolosmargenes.org/terapiaocupacional-economia-mercado-valemos-lo queremos-cobrar/ Emeric, D. (2015). Política, ideología y ejercicio profesional. Ocupando los márgenes, Recuperado de: http://ocupandolosmargenes. org/politica-ideologia-y-ejercicio-profesional/

Emeric, D. (2016). Terapeutas ocupacionales ¿Sin orgullo?: Ocupando los márgenes. Recuperado de: http://ocupandolosmargenes. org/terapeutas-ocupacionales-sin-orgullo/
Gajardo Jauregui, J., Catalán, C., Rioseco, R., \& Vildósola, I. (2018). Análisis de las prestaciones de terapia ocupacional incluidas en el régimen de garantías explícitas en salud (GES). Revista Chilena de Terapia Ocupacional, 17(2), 117-131. doi:10.5354/07195346.2018.48139.

Guajardo, A. Y Simo, S. (2010). Una terapia ocupacional basada en los derechos humanos. Revista de terapia ocupacional gallega (TOG, 7 (12), 1-25. Recuperado de: http://www.revistatog.com/ num $12 / \mathrm{pdfs} / \mathrm{maestros.pdf}$

Kielhofner, G. (2003) “Fundamentos Conceptuales de la Terapia Ocu-

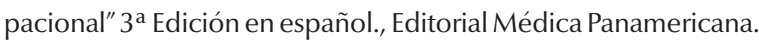

Kronenberg, F. (2007). Superar el apartheid ocupacional. En S. Simo Algado, F. Kronenberg, y N. Pollard. (Ed.), Terapia ocupacional sin fronteras. (pp.59-84) Madrid: Editorial Me dica Panamericana

Liborio, M.N. (2013) ¿Por qué hablar de salud colectiva? Revista Médica de Rosario, 79(3), 136-41. Recuperado de: http://www. saludcolectiva-unr.com.ar/docs/Liborio.pdf

López, C. (2018) ¿Qué es la parálisis cerebral en niños? Noticia de pagina web Universidad San Sebastián. https://www.uss.cl/ ciencias-salud/paralisis-cerebral-ninos/

Merino, C. (2016). Del proyecto interrumpido de la democratización de la salud a la práctica sanitaria neoliberal en Chile. 1960-1998. Osorno: Ediciones Universidad de Los Lagos.

Muñoz, C. (2014). La labor de la terapia ocupacional en el marco de los determinantes sociales de la salud en Chile. Revista Chilena de Terapia Ocupacional, 14(1), 73-80. doi:10.5354/0719-5346.2014.32391.

Olivares A., D., Morrison J., R., Yáñez R., R., \& Carrasco M., J. (2015). ¿Por qué puede ser difícil comprender qué es la Terapia Ocupacional? Una propuesta teórica desde cuatro perspectivas. Revista Chilena de Terapia Ocupacional, 15(1), 123-134. doi:10.5354/07195346.2015.37136

Organización mundial de la salud (2015). Salud y derechos humanos. Recuperado de: http://www.who.int/mediacentre/factsheets/ fs323/es/

Paredes, L., Vásquez, M., Muñoz, C. (2014). Praxis del Terapeuta ocupacional y su vínculo con la participación social: el ejercicio profesional en la realidad socio-sanitaria chilena. Revista de estudiantes de terapia ocupacional, 1 (2), 49 - 62 Recuperado de: http://www.reto.ubo.cl/index.php/reto/article/view/10

Pizarro, R. (2005). Desigualdad en Chile: desafio, económica y político. Polis, 4(10), 1-15.

Solsona, D. (2018). “Enseñar metodologías de investigación en la formación profesional" un dialogo interdisciplinario entre la sociología y la terapia ocupacional. RELMIS "Revista latinoamericana de metodología de investigación social", 15, 58-78.

Strauss, A. y Corbin, J. (2002) Bases de la Investigación Cualitativa. Técnicas y procedimientos para desarrollar la Teoría Fundamentada. Antioquia: U. de Antioquia 
Teletón. (2015). Parálisis Cerebral. Recuperado de: http://www.teleton.

cl/teleton/que-hacemos/rehabilitacion-integral/patologias/ paralisis-cerebral//

Tamayo, M., Besoaín, Á., \& Rebolledo, J. (2018). Determinantes sociales de la salud y discapacidad: actualizando el modelo de determinación. Gaceta Sanitaria, 32, 96-100.

Weitzman, M (2005) Terapias de Rehabilitación en Niños con o en riesgo de Parálisis Cerebral, Revista pediatría electrónica, (2) 1, 47-51. Recuperado de: http://www.revistapediatria.cl/volumenes/2005/vol2numl/8.html

Vilaboy Filgueiras, L; Vásquez, T.V. (2011). “Las actividades instrumentales de la vida diaria en personas con daño cerebral adquirido. Intervención de terapia ocupacional en un centro de autonomía personas. Revista de terapia ocupacional gallega (TOG), 8 (14), 1-7 Recuperado de: http://www.revistatog.com/num14/pdfs/ casol.pdf

Minsal. (Agosto de 2017). Ayudas Técnicas Marco Conceptualy Regulatorio hacia un Programa Nacional en Salud. Obtenido de https://www. minsal.cl/wp-content/uploads/2015/09/TO-Hernan-MeryAyudas-T\%C3\%A9cnicas.pdf 